\title{
Protée
}

\section{Raison et déraison dans la Lettre de Lord Chandos de Hofmannsthal (suite)}

\section{Claude Zilberberg}

Volume 29, numéro 2, 2001

Danse et altérité

URI : https://id.erudit.org/iderudit/030630ar

DOI : https://doi.org/10.7202/030630ar

Aller au sommaire du numéro

Éditeur(s)

Département des arts et lettres - Université du Québec à Chicoutimi

ISSN

0300-3523 (imprimé)

1708-2307 (numérique)

Découvrir la revue

Citer cet article

Zilberberg, C. (2001). Raison et déraison dans la Lettre de Lord Chandos de Hofmannsthal (suite). Protée, 29(2), 99-114. https://doi.org/10.7202/030630ar
Résumé de l'article

Cette étude se propose trois objectifs. En premier lieu, elle aborde le texte de Hofmannsthal d'une manière traditionnelle afin de montrer les limites de cette approche. En second lieu, elle s'efforce d'appliquer les hypothèses avancées par la sémiotique tensive (Fontanille-Zilberberg), hypothèses qui consistent pour l'essentiel dans la grammaticalisation de la circulation en discours des valences intensives et extensives. Enfin, une leçon émerge, nous semble-t-il, peu à peu, à savoir que l'affectivité ne se tient pas à la périphérie du sens, mais en son coeur même et, pour le dire sans précaution, l'affectivité devient la clef de la rationalité de ce type de discours, c'est-à-dire ce qu'il y a à comprendre dans l'acte de la lecture. 


\title{
RAISON ET DÉRAISON dans la L ettre de L ord C handos de H ofmannsthal (suite*)
}

\author{
Claude Zilberberg
}

N ote brève sur le miracle

Cetteanalytique del'augmentation et dela diminution, laquelle ne fait que transposer dans le plan du contenu le couple définitif [protase versus apodose], sera centrée, eu égard au tempo, sur la configuration de la «révélation» exprimée dansla séquencesuivante: «U n arrosoir, uneherse à l'abandon dans un champ, un chien au soleil, un cimetière misérable, un infirme, une petite mai son de paysans, tout cela peut devenir le réceptacle de mes révélations». C ette configuration, à la lecture du M icro-Robert 17 , joue, sous bénéfice d'inventaire, sur trois registres: (i) un registre cognitif opérant en discours le transfert de tel énoncéd'un émetteur jusquelà «silencieux» vers un récepteur «intéressé»; du point de vue morphologique, ce transfert suppose un passage du fermé vers l'ouvert; (ii) un registre cinétique exigeant que le transfert soit opéré avec «brusquerie» ou "soudaineté», c'est-à-dire à une très grande vitesse si on la compare à celles dont le sujet se sent capable ${ }^{18}$; il convient de préciser que l'effet de sens «vitesse» présuppose pour la cible d'abord, peut-être seulement, la syncope de toute attente, de toute actualisation, c'est-à-dire de toute préparation; (iii) un registre axiologique certain, sinon anagogi que, c'est-à-dire une quête des significations supérieures. Du point de vue épistémologique, nous ne pouvons pour l'instant qu'imaginer le concours des différentes composantes danstel effet de sens décisif pour un discours donné; ici l'hyperbole «miracle», admise par L ord C handos lui-mêmedans sa lettre, se laisse ainsi analyser:

$$
\text { «miracle» } \approx \text { [concession + soudaineté + anagogie] }
$$

Comme toute grandeur discursive, la configuration du «miracle» doit être caractérisée du point de vue paradigmatique et du point de vue syntagmatique. $\mathrm{N}$ ous limiterons l'approche paradigmatique du «miracle» aux définitions proposées par le M icro-Robert. Si nous rapprochons l'une de l'autre la première définition: «Fait extraordinaire où l'on croit reconnaître une intervention divine», et la troisième: «C hose étonnante et admirable qui se produit contre toute attente», il apparaît que leur différencerenvoieà la distinction proposée par $D$ urkheim dans Les Formes élémentaires de la vie religieuse entre le religieux et le divin :

Ce que nous trouvons à l'origine et à la base de la pensée religieuse, ce ne sont pas des objets ou des êtres déterminés et distincts qui possèdent par eux-mêmes un caractère sacré; maisce sont des pouvoirsindéfinis, desforces anonymes, plus ou moinsnombreusesselon lessociééé, parfoi smêmeramenées à l'unité, et dont l'impersonnali itéest strictement comparable à celle des forces physiques dont les sciences de la nature étudient les manifestations. 19

La première définition du M icro-Robert renvoieau divin, la seconde au religieux, le divin présupposant le religieux, même si le divin refuse, par principe, de reconnaître sa dette à l'égard du religieux. Le mérite de cette distinction réside dans le refus du syncrétisme, souvent admis, de ces deux notions et dans son corollaire, à savoir la nécessité de penser le religieux indépendamment de et commeanté rieurement au divin. D u point de vue syntagmatique, infiniment plus délicat, le «miracle» relève d'une sémiotique 
de l'événement, ayant pour assiette le survenir et son improbabilitéirréductible, puisquela forceillocutoire del'affirmation du «miracle» tient précisément au fait que la réalisation n'ait rien à voir avec I'«ordre [admis] des choses» 20 ; si l'on suspend, si l'on retranche dela profession du «miracle» les pourtant, les en dépit de, les malgré, les bien que qui le modalisent, c'en est fini du «miracle» luimême; le «miracle» n'existe que pour celui qui l'accueille sans la moindre réserve mentale, qui montreà son égard la même adhésion que celle que le positiviste le plus endurci professe pour le déterminisme. N ous interpréterons la profession du «miracle» comme dépassement de la «nature» au plan figuratif et comme concession au plan figural, c'està-dire comme dépassement de la causali ité reçue.

Si nous convoquons la définition que donne le M icroRobert dela soudaineté: «Q ui arrive, seproduit en très peu de temps», nous observons qu'ellefait sens dans une sémiotiquefigurative del'événement: quelquechose «arrive, seproduit», puis dans une sémiotique de la temporalité: «en très peu de temps». M aisc'est peut-êtred'une sémiotique générale de l'intervalle que la soudaineté en discours reçoit sa pleine signification: la soudaineté dénote une accélération laquelle, par structure, abrège la durée et la réduit à l'intervalle le plus bref que nous ayons le sentiment de saisir: I'«instant»- défini, sinon défendu, semble-t-il, par son indivisibilité de fait; la soudaineté signifie assurément que le temps de passage d'un état à un autretend «anormalement» vers la nullité 21 mais cette dernière, loin d'être pure déception, s'avère au contraire mobilisatrice: certes, elle déstabilise le sujet, le malmène, le «pousseà bout» mais, ce faisant, elle fait sentir au sujet l'urgence de sa propre récollection. N ous admettrons que la soudaineté, aussi longtemps qu'elle demeure non résolue, intervient ici au titre de dépassement, d'exorbitance de la durée.

Enfin l'anagogie, ignorée du M icro-Robert, est définie en cestermes par Le G rand Robert: «Élévation, ravissement de l'âme dans la contemplation mystique. V. Extase. Recherche du sens mystique del'Écriture». N ous enregistrons tout d'abord un syncrétisme du sentir et du savoir, mais du point de vuesémiotique, on doit supposer unemodification de la fonction sémiotique descriptible comme substitution d'un signifié jusque-là potentialisé au signifié manifesté. Un «arrosoir» peut devenir le «réceptacle [d'une] révélation ». Là encore, pour faire court, nous admettrons êtreen présence d'un dépassement de la lettre.
La configuration du «miracle» se révèle singulièrement cohérente puisque ses composantes, sous bénéfice d'inventaire, présentent la même structure tensive.

\begin{tabular}{|c|c|}
\hline traits sémantiques & définitions tensives \\
\hline «miracle» & $\begin{array}{c}\text { en tant que } \\
\text { dépassement de la «nature» }\end{array}$ \\
\hline concession & $\begin{array}{c}\text { en tant que } \\
\text { dépassement de la causalité }\end{array}$ \\
\hline soudaineté & $\begin{array}{c}\text { en tant que } \\
\text { dépassement de la durée }\end{array}$ \\
\hline anagogie & $\begin{array}{c}\text { en tant que } \\
\text { dépassement de la lettre }\end{array}$ \\
\hline
\end{tabular}

Chaque composante manifeste, projette dans le registre qui est lesien, un excès, c'est-à-dire que, sel on la sémiotique de l'intervalle que nous préconisons, elle change, ici sublime, une limite en degré, avec les corrélats attendus: le «miracle»échappeà la «nature» et à la causalité, sinon àla contention qui I'astreint; la temporalité échappe à l'uniformité; le sens échappe à sa clôture.

Cette analyse du «miracle» doit être maintenant rattachée à la sémiotique de la force. La configuration du «miracle» intéresse la manifestation, le paraître de l'être, toutefois dans la mesure où le «miracle» se répète, encore une fois de manière aléatoire, dans la mesure où il est potentialisé, mis en mémoire, ainsi que Lord Chandosl'explique dans le passage suivant:

Quel rapport y a-t-il, en effet, avec la pitié; quel rapport avec un enchaînement d'idées humaines, naturelles, dans cette aventure d'un autre soir : jetrouve sous un noyer un arrosoir à moitiéplein qu'un jeunejardinier a oubliélà, et cet arrosoir avec l'eau qui est dedans, obscurcie par l'ombre de l'arbre, avec un scarabée allant d'un bord à l'autre à la surface de cette eau sombre, cetteconjoncture de donnéesfutilesm'expose à unetelle présence de l'infini, metraversant de la racinedes cheveux à la basedestalons, quej'ai envie d'éclater en paroles dont je sais, les eussé je trouvées qu'elles auraient terrassé ces chérubinsauxquels jene crois pas; jeme détourne ensuite de celieu, en silence, puis, dessemaines plustard, apercevant ce noyer, passe près de lui avec un timide regard de côté pour ne pas effaroucher le sentiment laissé par le mi racle qui souffle là, autour du tronc, ne pas chasser ces frissons autres que terrestres pesant encore dans le vois nage de ces broussailles.

N ous admettrons que le «miracle» devient la variable temporelle discontinue d'une fonction continue: la «pré sence de l'infini ». D ans ces conditions, l'être lui-même se 
présente comme un terme neutre: [ni augmentation $+\mathrm{ni}$ diminution], de sorte quela manifestation est seule susceptible d'augmentation sous les deux modalités suivantes: (i) l'avènement, c'est-à-dire le passage «inouï » de [0] à [1]; (ii) l'itération, la répétition à intervalles non réguliers, et non cumulative - afin de laisser à l'attente son acuité - de cette donation de la «présence de l'infini ».

\section{La composante tonique}

Abordons la seconde sous-dimension de l'intensité, à savoir la tonicité. Si le traitement sémiotique du tempo intéresse prioritairement la temporalité en y déterminant comme des «creux», des béances temporelles, commesi le sujet était rejeté ou expulsé hors du temps courant, la tonicité semble surtout affecter, en quelque sens qu'on l'entende, la spatialité, c'est-à-dire la distribution des espaces. Si nous proposons d'emblée cette commutation, c'est que tout indique que l'espace pour le vivant est un espace dé coupé, parcellisé, justiciable d'une «carte» et ordonné par les possibilités, les attirances, mais également les menaces et les interdits relatifs à la circulation entre ces espaces.

Un processus d'accroissement peut être simple ou complexes'il prévoit aussi un programme visant à prévenir la diminution. La formule sémiotique de l'accroissement serait une «variété» de la formule générale:

[programme] + [contre(contreprogramme)]

L'être étant plénitude et le paraître déficit, Lord C handos va assumer la double tâche d'excéder la plénitude, en concordance avec la centralité de l'excès que nous avons invoquée au début de ce travail, et d'éviter à la manifestation toute dégradation supplémentaire. C'est le second point qui nous retiendra d'abord.

D ans le discours de Lord Chandos - et c'est l'une des étrangetés les plus remarquables de ce texte qui pourtant n'en manque pas-, l'espace comme étendue est virtualisé, et l'effectuation de cette opération ne lai sse subsister que des points d'ancrage, au demeurant séparés les uns des autres. $M$ ais ces points d'ancrage sont traités comme des volumes, demêmeque précédemment la duréese présentait comme un inventai re demoments. En effet, une des voies qu'emprunte dans cet univers de discours la tonicité pour sémiotiser l'espace consiste justement en la conversion de tout objet en capacité de contenance, en «réceptacle» de la «présence»:
Bien plus, à la représentation précise d'un objet absent peut échoir en partage ce destin incompréhensible d'être emplie jusqu'au bord du flux doux et brutal de ce sentiment divin.

La première configuration que nous en registrons est donc aspectuelle, puisqu'elle porte sur le remplissement d'un volume. $M$ ais au-delà il est permis de penser que cette virtualisation des surfaces et ce creusement des volumes sont au service de la tonicité, puisque cette dissymétrie est conforme au partage entre des val eurs d'absolu concentrées et toniques et des valeurs d'univers atones et diffuses, partage que nous dicte notre imaginaire:

\begin{tabular}{|c|c|c|}
\hline signifiant & surface & volume \\
signifié & $\begin{array}{c}\text { diffusion } \\
\text { [propre aux valeurs } \\
\text { d'univers] }\end{array}$ & $\begin{array}{c}\text { concentration } \\
\text { [propre aux valeurs } \\
\text { d'absolu] }\end{array}$ \\
\hline
\end{tabular}

D emêmequela durée, l'étoffe du tempsest défaiteau profit d'une succession rapsodique, arythmique d'instants extatiques, de mêmela texture del'espace est abolie et les pleins fortuits du monde d'aujourd'hui sont comme autant de trous dans le monde d'autrefois.

La seconde configuration identifiable est celle bien connue des «vases communicants» et se présente comme un corollaire de la transformation des surfaces en contenants sur le plan de l'expression et de la saturation de la tonicité au titre de programme sur le plan du contenu. Le théorème sémiotique à peine implicite affirme l'indivisibilité del'éclat, si bien quece dernier peut certes occuper, visiter des places diverses, ce que ne cesse d'affirmer Lord C handos, mais successivement, jamais simultanément. D e là le recours si fréquent dans ce texte à l'énumération de grandeurs hétéroclites entreelles. T outegrandeur, du fait même qu'elle est signifiée comme contenance, est apte à devenir un «réceptacle» del'éclat, et lemoi deLord Chandos prend place dans cette mise en paradigme qui, loin de précéder la syntaxe, en procède au contraire. L'épisode du «poison pour les rats» est exemplaire à cet égard:

Ainsi, récemment, j'avais donnéordredeverser en abondance du poison pour les rats dans les caves à lait d'une de mes métai ries. Verslesoir, je sortisà cheval sans plussonger, comme vousle présumez, à cettehistoire. Alors, tandisquemon cheval avance au pas dans la haute terre d'un champ retourné et que je découvre rien de plus inquiétant à proximité de moi 
qu'une couvée de cailles apeurées et au loin, au-dessus de l'ondulation des labours qu'un grand soleil couchant, alors s'ouvre au fond de moi cette cave emplie par l'agonie d'un peuple de rats. Tout était au-dedans de moi : [... ].

Lord Chandos indique lui-même que le «principe d'individualité» cher à $\mathrm{N}$ ietzsche est mis à mal et fait état d' «une participation contre nature, [d']une intrusion au-dedans de ces créatures, ou lesentiment qu'un fluide de vieet de mort, de rêve et de veille s'est écoulé en elles l'espace d'un instant - d'où venu?». C esdeux procédés permettent aux moments intenses de la manifestation de conserver justement leur pleine «efficience» (C assirer), ce qui est un des problèmes de sémiotique générale: comment fair reéchec à la dissipation de toute tonicité advenue? Comment faire échec à la temporalisation diminutive de l'éclat?

$M$ ais ces quanta de «présence infinie», dont la haute concentration est préservée par la morphologie spécial e des grandeurs qui les recueillent, subissent des processus d'augmentation, ici effrayants. Ainsi l'«agonie [du] peuple de rats» va faire l'objet de deux accroissements distincts. Le premier advient par convocation d'une hypotypose rendue possible par la communauté des références entre l'énonciateur et l'énonciataire: «V ous vous souvenez, ami, avec quel art Tite-Live évoque les heures qui précédèrent la destruction d'Albe-la-Longue?». M ais ce rappel permet à l'énonciateur d'enchaîner un comparatif et un superlatif I'un comme l'autre exorbitants: «[... ] mais c'était plus encore, c'était plus divin, plus bestial ; et c'était du présent, le présent le plus plein, le plus sublime». N ous sommes au cœur même del'excès, puisquece dernier admet ici cequ'il exclut en principe: un nouvel excès! T out se passe comme si le pléonasmelui-même pêchait par insuffisance radicale.

Le second procédé, I'hyperbole, se distingue de I'hypotypose en ce sens qu'elle porte sur la spatialité et I'hypotypose sur la temporalité. Tandis que D umarsais dans son Traité des tropes manifeste une antipathie certaine pour I'hyperbole, qu'il juge contraire à I' «esprit français», Fontanier dans Les Figures du discours avance une définition étonnante à bien des égards:

L'hyperbol eaugmente ou diminueles choses avec excès, et les présente bien au-dessus ou bien au-dessous de ce qu'elles sont, dansla vue, non detromper, maisd'amener à la véritémême, et de fixer, par ce qu'elle dit d'incroyable, ce qu'il faut réellement croire. 22
Ce qui est étonnant, c'est de voir Fontanier proposer comme critère de la vérité, non l'adéquation, non la référence plus ou moins directe à un consensus ou, à l'inverse une justesse transcendante inscrite dans les choses mêmes, mais bien l'excès lui-même! D ès lors que l'affect est prioritairement défini par sa démesure vécue, tout se passe comme si, dans le cas de la sémiotique de l'événement, la grande affaire était la quantité et non la qualité; le risque pour l'énonciateur n'est pas, comme on dit couramment, «d'en faire trop», mais au contraire «pas assez», de ne «pas se montrer à la hauteur » des attentes, parfois suspectes, de l'énonciataire. Tout se passe comme si la prédication de I'«incroyable» était affaire non de démonstration, mais seulement de témoignage et d'empathie perlocutoire.

D e même que l'hypotypose opérée par Lord C handos fusionne deux temporalités «distantes» I'une de l'autre, celle de l'énonciateur et celle de T ite-Live «[évoquant] les heures qui précédèrent la destruction d'Albe-la-Longue», I'hyperbole conduisant à un accroissement de la tonicité semble obtenue par la fusion, le raccordement de deux espaces hétérotopes: celui de la «mèrequi sentait tressaillir autour d'elle ses petits mourant», et celui dont tout énonciateur s'institue le centre:

S'il s'est trouvé un esclave pour voir, saisi d'impuissante horreur, N iobé changé en pierre, celui-là a dû traverser ce quej'ai traversé quand en moi l'âme de cet animal montra les dents au destin monstrueux. 23

La psychanalyse connaît ces advenir sous lesnoms d' «incorporation » et d'«introjection ». À cesujet, Laplanche et Pontalis écrivent:

II convient de maintenir une distinction, [...] entre incorporation et introjection. En psychanalyse la limite corporelle est le prototype de toute séparation entre un intérieur et un extérieur; leprocessusd'incorporation serapporteexplicitement à cette enveloppe corporelle. Le terme d'introjection est plus large: ce n'est plus seulement l'intérieur du corps qui est en cause, maisl'intérieur del'appareil psychique, d'uneinstance, etc. $[\ldots]^{24}$

Si la psychanalyse est portée à faire dériver I'«introjection » de l'«incorporation», la sémiotique et la phénoménologie conçoivent plutôt la seconde comme un cas particulier de la première. En effet, dans La Catégorie des cas, $\mathrm{H}$ jelmslev évoque au titre de seconde dimension le «degré d'intimité 
avec lequel deux objets sont liés ensemble» 25 , de sorteque I'«introjection » et I' «incorporation » psychiques entreraient dans la dépendance de l'«intimité» sémiotique. $M$ ais du point de vue tensif, le point important est le suivant: il s'agit de corréler les morphologies flagrantes de l'extensité aux destins possibles de l'intensité. En définissant l'«inhérence» comme le fait pour la grandeur $[A]$ de se trouver à l'intérieur de la grandeur $[B]$ et le «non-contact» comme lefait pour les grandeurs $[A]$ et $[B]$ de demeurer extérieures I'une à l'autre, on peut avancer I'hypothèse selon laquelle le progrès du «non-contact» vers I'«inhérence» est corrélé à celui du passage de l'atonie à la tonicité, soit:

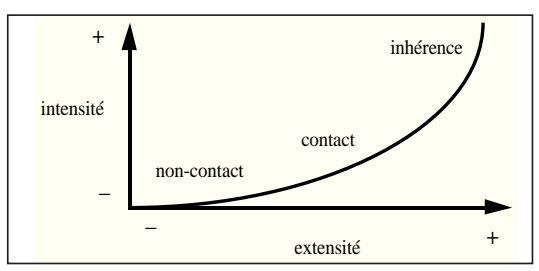

Si cette corrélation - une corrélation n'étant qu'une suite dénombrable de commutations remarquables- est attestée, elle signifie que le raccordement des espaces sur le plan de I'expression aurait pour contrepartiesur leplan du contenu une addition, une composition, enfin une sublimation des valences toniquessi le résultat est éprouvé comme supérieur à la simple somme arithmétique de ces mêmes valences. Si la reconnaissance de l'hyperbole sur le plan de l'expression semble aisée, sa compréhension sur le plan du contenu semble promettre des difficultés considérables.

Le traitement de la tonicité dans le texte de $\mathrm{H}$ ofmannsthal nous propose donc une alternance paradigmatique embarrassante: tantôt les phases de manifestation se succèdent sans communiquer les unes avec les autres, sans que leurs valences toniques s'ajoutent les unes aux autres; leur mérite consistealors dans leur non-diminution ; tantôt, comme dans l'épisode du «poison pour les rats», le texte nous invite à sentir que les valences toniques, ici par hypotypose et par «introjection», fusionnent les unes avec les autres et établissent, pour ainsi dire, «à vue» une tonicité supérieure. U n peu plus loin, Lord Chandos «lisse»la démesure del'épisode du «poison pour les rats»: «[...] jesens un affrontement délicieux, tout simplement infini, en moi et autour de moi, et parmi les matières qui s'affrontent il n'en est aucune dans laquelle je ne puisse me glisser». Au passage, il convient de remarquer que, sur ce point, $\mathrm{H}$ of- mannsthal prend, sans doute à son insu, le contre-pied de la psychanalyse puisque la doxa freudienne lie en principe I'introjection à l'euphorie et la projection à la dysphorie.

\section{Progression implicative et progression concessive}

Letraitement del'accroissement et de la diminution peut être restreint au couple [ajout vs retrait] et à la linéarité «scolaire»qu'il projette. M aisle discours mérite, nous semblet-il, mieux. L'implication et la concession, mais dans une moindre mesure, ont été depuis reconnues par Fontanille et Zilberberg comme opérateurs conceptuels de premier rang, tantôt pour souligner la nécessité d'une relation stable de dépendanceentre deux grandeurs, tantôt l'arbi traire de cette même relation; autrement dit, l'implication et la concession ont été réservées à l'extensité admise comme théâtre des coexistences effectives, mais il n'y a là sans doute qu'un usage, lequel, à l'instar de tous les usages, aveugle. La sémiotique del'intervalle, aussi bien par ses données que par ses ressorts, interviendrait en intensité comme extensité et permettrait dediscriminer, des styles ascendants distincts, des «manières» de croitre ou de s'accroître sous le regard d'un observateur averti et attentif. M oyennant ces prémisses, nous distinguons une progression implicative concordant avec les attentes du sujet et une progression concessiveselon la surprise. Elles s'opposent l'une à l'autre à un double titre: (i) au titre de l'intensité, c'est-à-dire du vécu de l'affect, la progression implicative proscrit l'excès et recommande le non-dépassement, tandis que la progression concessive a pour objet de quêtela démesure; (ii) au titre de l'extensité, c'est-à-dire du rapport naïvement métrique posé entre la taille de l'antécédent et celle du conséquent; de deux choses I'une: ou bien le sujet a le sentiment d'une proportion heureuse, d'une convenance, ou bien celui d'unenette, souvent choquante, disproportion; dans ce dernier cas, la petitesse de l'objet rapportée à la démesure des conséquences qu'il contrôle est résolue par la concession selon le modèle familier: «petite cause, grands effets» 26 . Soit:

\begin{tabular}{|c|c|c|}
\hline & progression implicative & progression concessive \\
\hline $\begin{array}{c}\text { esthésie intensive } \\
\text { dépassement vs non-dépassement }\end{array}$ & nelon l'attente & selon la surprise \\
\hline $\begin{array}{c}\text { esthésie extensive } \\
\text { proportion vs disproportion }\end{array}$ & proportion & dépassement \\
\hline
\end{tabular}

Et c'est bien ainsi que Lord Chandos l'entend luimême: 
Chacun de ces objets, et mille autres semblables dont un œil ordinaire se détourne avec une indifférence évidente, peut prendre pour moi soudain, en un moment qu'il n'est nullement en mon pouvoir de provoquer, un caractèresublime et si émouvant, que tous les mots pour le traduire, me parai ssent trop pauvres.

N ous sommes très proches du déplacement 27 freudien si cen'est que nous sommes ici dans la veille et que le déplacement est donnépar F reud commeun mécanismedu rêve. À moins qu'il ne faille renverser les termes de la problé matique: I'aboutissant d'une progression, notamment du fait de la précipitation qui l'emporte, aboutit à une accentuation qui confèreà la grandeur qu'ellefrappece que Lord Chandos appelle «un caractère sublimeet si émouvant, que tous les mots pour le traduire, me parai ssent trop pauvres». $D$ ans ces conditions, cen'est pas le déplacement qui éclaire l'accent, mais bien l'accent qui, dégagé desa phonétisation, éclairele déplacement pour autant queles valences toniques au titre de contenu ont vocation à circuler, de sorte que leur fixation à telle saillance du plan de l'expression est à jamais provisoire. $D$ ans lemêmeesprit, nous savons, depuis La Grammaire des fautes d'H . Frei, que l'autorité d'une grammaire, ici même une grammaire discursive en projet, se manifeste par les fautes qu'elle fait connaître au sujet discourant. À cet égard, une des fautes les plus lourdes que la grammaire discursive est en mesure de découvrir au sujet consiste à demander, à expecter d'une progression implicative ce qui ne peut être accordé que par une progression concessive. Cequi fait sans doutela «gravité» de cettefaute, c'est la connivence entre la distinction paradigmatiqueentre valeurs d'absolu et valeurs d'univers et la distinction syntagmatique entre progression concessive et progression implicative. Soit:

\begin{tabular}{|c|c|c|}
\hline paradigme & $\begin{array}{c}\text { valeur } \\
\text { d'absolu }\end{array}$ & $\begin{array}{c}\text { valeur } \\
\text { d'univers }\end{array}$ \\
\hline$\checkmark$ & $\begin{array}{c}\text { progression } \\
\text { concessive }\end{array}$ & $\begin{array}{c}\text { progression } \\
\text { implicative }\end{array}$ \\
\hline
\end{tabular}

En un sens restreint, la Lettre de L ord C handos s'avère un texteinitiatique dans la mesure où lesurvenir improbable de l'accent est accepté et assumé comme la forme de vie désormais exclusive:

En de tels instants, une créature sans valeur, un chien, un rat, un insecte, un pommier rabougri, un chemin de terre tortueux escaladant la colline, un caillou couvert de mousse comptent pour moi davantage que n'a jamais fait l'amante la plusbelle, la plusprodiguedela plusheureusedemesnuits.

Cette forme de vie s'avère excentrique eu égard à celle associant auparavant la culture admirée et les privilèges crus définitifs de la naissance. $M$ ais, comme en vertu d'un renversement vertigineux, les vécus de L ord $C$ handos sont en parfaite résonance avec l'évocation par $C$ assirer des «dieux de l'instant» sel on U sener:

Q uand la sensation instantané attribue à la chose devant nous, à l'état dans lequel nous nous trouvons, à l'action dela forcequi nous surprend, la valeur et en quelque sortel'accent du divin - alorsle dieu de l'instant est ressenti et crée. II est devant nous dans sa particularité et son unici té immédiates; non comme la partie d'une force qui peut se révéler ici et là, dans divers lieux de l'espace, à divers moments et dans différentssujets, se révéler dediversesmanièrestout en restant la même, mais comme quelque chose qui n'est présent qu'ici et maintenant, dans ce moment unique et indécomposable du vécu, pour cesujet uniquequ'il envahit decettesienne présence et tient sous son charme. 28

Une concordance aussi profonde disqualifie, nous semble-til, la question de savoir si, selon un terme expédient, Lord Chandosest «fou » ou non: il nel'est pas. T out au plus, pourrait-on dire, qu'il l'est tout autant qu'un hommequi vivrait à la lettre ce que ceux qui l'entourent vivraient au figuré, tout autant qu'un grand mystique égaré au milieu d'un peuple unanime de voltai riens ricanants - ou l'inverse bien évidemment. Lord Chandos est sur ce point tout à fait lucide:

Je fus envahi, au milieu d'elles, [les «idées» de Sénèque et de Cicéron] par le sentiment d'une terrible solitude; je me fisl'effet dequelqu'un qui serait enfermédansun jardin empli rien que destatues dépourvues d'yeux; je m'enfuisà nouveau en pleine campagne.

$M$ ais ces vicissitudes sont du ressort de l'extensité, c'est-àdire d'une décision concernant les tris et les mélanges, et leurs aboutissants, les espaces de rangement accueillant les grandeurs mondaines.

L'introspection conduite par Lord Chandos n'est pas sans mérite puisqu'elle exhibe la présupposition réciproque, ou la concordance, existant entrel'accentuation comme localité remarquable et la concession comme parcours sai- 
sissant: «C escréatures muettes et parfoisinaniméess'élancent vers moi avec un amour si entier, si présent, que mon regard combléne peut tomber al entour sur aucunesurface morte». Si nous convenons de banaliser un instant le «raisonnement» de L ord C handos, nous pouvons le présenter ainsi: bien que telle grandeur du contenu vaille dans le monde d'autrefoiscommenulle, elle peut surgir, sanstitreaucun, sans la moindre justification, comme suprême dans le monde d'aujourd'hui - simplement par synecdoque, par délégation de l'immanence indemne de la «présence».

Sans traiter la question comme elle le mérite, nous admettons que la Lettre de Lord Chandos est en résonance avec bien des pages du second volume de L a Philosophiedes formes symboliques de C assirer. $N$ 'étai ent les conventions et les contraintes inhérentes au genre littéraire choisi par $\mathrm{H}$ ofmannsthal, Lord Chandos pourrait, selon nous, aisé ment donner son acquiescement à ces autres lignes de Cassirer:

Lemythes'en tient exclusivement à la présencede son objet, à l'intensité avec laquelle celui-ci assaille la conscience à un instant déterminéet prend possession d'Ale. [...] La conscience est prisonnière del'impression, de la simplicitéde son êtrelà, elle n'a ni le désir ni la possibilité de juger ce qui est donné hic et nunc, de lecritiquer et dele borner dansson objectivité en le mesurant à ce qui n'est pas donné, à un terme passé ou futur. [...] L'image dela réalitéqui naît de cette manière est pour ains dire privée de sa troisième dimension [...] ${ }^{29}$

À l'instar de Rousseau dans Les Rêveries éprouvant la suffisance, sinon la «perfection » du «sentiment de l'existence dépouillé de toute autre affection $\gg 30$, Lord C handos tient, comme obligépar la qual itééminentede son correspondant, à formuler l'indicible: «[...] nous pourrions entrer dans un rapport nouveau, mystérieux avec toute l'existence, si nous nous mettions à penser avec le cœur».

Cette dernière remarque, que Pascal aurait pu signer, appelle un bref commentaire. Si I'on adopte la lecture de $\mathrm{H}$ eidegger proposée par $\mathrm{G}$. Steiner, il est clair que la L ettre de L ord C handos fait entendre, avant la date, des accents heideggeriens. G. Steiner écrit notamment:

Lorsque la pensée est présente au plus profond de l'homme, elle [...] implique ce que le grand mystique $M$ aître $E$ ckhart appelait das Seelenfunklein, la «flammèchedel'âme» - $\mathrm{H}$ èdegger l'appellera le «coeur». Encore une fois, nous sommes appelés à suivre l'étymol ogie (le langage sait toujours mieux quenous). Cor, corids, le «coeur », et au centre du procès ou acte de se «ra-corder» qui inspire et illumine le penser authentique. $[\ldots]^{31}$

Parce qu'il a vécu dans I'«oubli de l'être», Lord Chandos «connaît»l'aletheia, cette tension entre (se) montrer et (se) cacher, entre (se) montrer par le fait même de (se) cacher, mais à la différence du «dernier» $\mathrm{H}$ eidegger, s'effaçant derrière $\mathrm{H}$ ölderlin, Lord Chandos, s'il a conscience d'accéder à la Lichtung, affirme en même temps être privé des mots pour le dire, puisquele langagetel qu'il l'a penséet pratiqué jusqu'à maintenant est aliénant, étranger, sourd à la «pré sence». $M$ ais les privilèges de la fiction sont tels que $\mathrm{H}$ ofmannsthal a trouvé lui les mots dont Lord Chandos a cru devoir constater que désormais ils le fuient. C'est dire que $\mathrm{H}$ ofmannsthal a accédé pour ce texte à ce que $\mathrm{H}$ ei degger a seulement pressenti :

Car la pensée (das D enken), dans son dire, porte seulement au langage la parole inexprimée de l'Être.

La tournure ici employée: "Zur Sprache bringen», «porter au langage» est désormaisà prendre en son sens littéral. 32

L'accès à ce que l'on appelle la «création artistique», ici refusé, là accordé, refusé à L ord C handos et, semble-t-il, à $\mathrm{H}$ eidegger, accordé à $\mathrm{H}$ ofmannsthal, devient la pierre de touche, pour autant que l'art ne consiste pas à dire, mais à laisser dire en se voulant d'abord à l'écoute, comme si en cette affaire l'attention était première, renvoyait à ce que M erleau-Ponty appelle dans L'E $\mathcal{E}$ il et I'Esprit tantôt un «mystère de passivité», tantôt un «secret de préexistence», et «le reste», à savoir l'invention, l'originalité dans le traitement du sujet, les contraintes ou les routines d'un genre... secondaire.

\section{D es actants sous influence}

Les actants, indépendamment de leur constitution théorique plus ou moins fortuite (Propp, Tesnière, Souriau, G reimas), sont définis par leur engagement dans la narrativitésur leplan figuratif, et sur leplan figural par l'inflexion polémique, adversative attribuée par G reimas aux structures profondes 33 . D epuis, trois données ont retenu l'attention: I'hypothèse du schématisme tensif, résumée par la «coalescence» de l'intensité, le sensible, et de l'extensité, l'intelligible; la nécessité d'une sémiotique de l'intervalle en tant que clef d'accès à l'ascendance et à la décadence tensives; 
enfin la tension prometteuse entre syntaxe implicative et syntaxe concessive. Cestrois directions sémantiques ne sont pas sans conséquence pour l'actant. Trop succinctement sans doute, deux points se détachent: (i) la dualité des dimensions intensiveet extensivese traduit pour l'actant sujet par une relative spécialisation: eu égard àl'intensité, le sujet est un sujet sensible, sentant, soucieux de mesurer ce qu'il ressent, donc plutôt un patient; eu égard à l'extensité, le sujet, dans la mesure où il opère, incessamment et souvent à son corps défendant, par tris et mélanges, plutôt un agent; (ii) les valences et les sub-valences assurent une précieuse transition entre les dimensions ordonnant l'espace tensif en sa globalité et les actants définis par leur adresse, leur local ité, leur quelque part dans ce même espace tensif.

\section{Distensions du sujet}

Ce qui fait problème, c'est, nous semble-t-il, la délicatesse du sujet telle qu'elleressort de ces pages. Le sujet est inscrit dans l'espace tensif qu'il projette, si bien que les catégories tensives, à moins que la légitimité de cette exception ne soit dûment justifiée, s'appliquent aussi au sujet. En concordance avec la schizie de la tensivité en [intensité versus extensité], nous scindons le sujet en sujet sensibleselon l'intensité et en sujet praxiqueselon l'extensité, le premier préoccupé d'abord de ce qu'il ressent, le second d'abord de ce qu'il fait et défait:

\section{[sujet sensible versus sujet praxique]}

Si nous considérons le sujet sensible en le rattachant aux valences extrêmes [éclatant versusfaible], nous accédons au couple:

$$
\text { [sujet extatique versus sujet équanime] }
$$

Ces sujets ne sont pas définis par une position, mais par leur statut structural : le sujet extatique obéit à la complexité structurale: [éclat + faiblesse] et se présente en discours comme un sujet selon l'alternance [tantôt..., tantôt...], comme il le remarque lui-même: «M ais quand cet étrange enchantement m'abandonne, je ne sais plus rien dire à son sujet [...]». D e son côté, le sujet équanime est plutôt un sujet selon la neutralitéstructurale: [ni éclat + ni faiblesse]; Lord Chandos, pour désigner cette complexion, parle d'«engourdissement de son être», mais le point est malaisé puisque si ces deux régimes de val ences ne sauraient communiquer l'un avec l'autre, néanmoins chacun peut devenir pour l'autre un point de vue. Ces sujets sont donc définis par la valeur de l'intervalle qu'ils parcourent, qu'ils «discourent». Rousseau, à partir des extraits que nous avons produits, est un bon représentant du sujet équanime, défini en priorité par le jeu de ses valences tensives, même si un syncrétisme, à démêler, superpose la bassesse des valences de tonicité et leur localisation dans I'espace tensif:

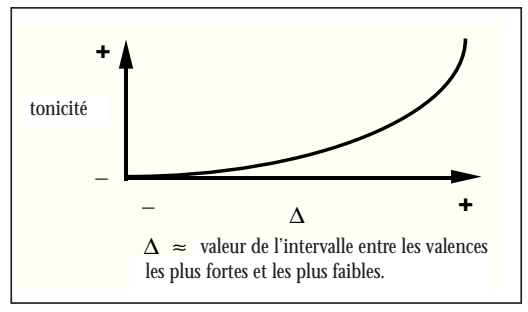

Si cette corrélation était vérifiée par ailleurs, elle signifierait que les écarts propres à la «cyclothymie», dont tout un chacun est susceptible, augmentent à mesure quel'on s'approche des valences les plus hautes de la thymie - ce qui n'est pas, loin s'en faut, contre-intuitif.

Portrait du sujet extatique

II nous incombe maintenant d'aborder la description du sujet extatique, puisque nous avons admis que c'est de cette possibilitéque Lord C handosse montre dans lemonde d'aujourd'hui leplus proche. L'affaireest simpleet malaisée à la fois: elleest simpleen cesens quele sujet doit reproduire lepartage dela dimension del'intensitéen sous- dimensions: [tempo versus tonicité]

elle est malaisée en ce sens que ce clivage du sujet en: [sujet sidéré versus sujet tonalisé]

n'est pas conformeà nos habitudes, à nos routines mentales. $\mathrm{N}$ ous avons retenu le terme de sidéré d'abord en raison de sa définition selon Le M icro-Robert: "Sidérer. v. tr. Fam. Frapper de stupeur. Cette nouvelle m'a sidéré». Le Littré ignoreleverbe «sidérer», maismentionnelesubstantif «sidé ration $»$ :

1. Terme d'astrologie. Influence subite attribuée à un astre, sur la vie ou la santé d'une personne. 2. Termede médecine. État d'anéantissement subit produit par certaines maladies, qui semblent frapper lesorganesavec la promptitudedel'édair ou de la foudre, commel'apoplexie; état autrefois attribuéà I'influence malfai sante des astres. 
Le Grand Robert modernise et dramatise la définition du Littré:

1. Astrol. Action subitedel'influenced'un astre sur la vie, la santéd'une personne. 2. M éd. Anéantissement subit desforces vitales avec état de mort apparente (notamment sous l'effet d'un agent électrique). Sidération del'apoplexie, d'unegrave dépression mentale.

En intensité, le verbe «sidérer » comporte à l'évidence une valence de soudainetéqui est l'humaine limite de la célérité, c'est-à-dire que le sujet, incapable d'entrer en synchronie avec le survenir, s'arrête! ${ }^{34}$ et mesure une précipitation qui pour lui-même a valeur de recul: «Influence subite... État d'anéantissement subit... Action subite... A néantissement subit... ». L'existenceest ainsi à la merci du tempo, ou encore, ainsi que l'indique Valéry, le tempo s'impose comme le «facteur d'existence»35 - ou d'inexistence!

Ceci n'est pas sans conséquence pour l'extensité, c'està-direle regroupement conventionnel, ou occasionnel, des grandeurs en classes; au titre du pouvoir-faire qu'on leur accorde, l'astrologieet la médecine appartiennent à la même classe de grandeurs, parce qu'elles présentent la même identité valencielle, ici l'attribution - apparemment divinisante- del'instantanéitéau pouvoir-faire propreà certains agents, qu'ils soient supra ou infra-humains. Un point mérite d'être relevé. L'appartenance detelles grandeursà la même classe permet au Littré d'assimiler, sans plus de précautions, les vitesses des actants de contrôle (Fontanille) puisque, de certaines maladies, il affirme qu'elles «semblent frapper les organes avec la promptitude de l'éclair ou de la foudre» mais, de son côté, D urkheim admet le même glissement:

Un individu entret-il en contact avec elles [les forces impersonnelles des cultes totémiques] sans avoir pris les pré cautionsconvenables? II en reçoit un choc quel'on a pu comparer à l'effet d'une décharge électrique. 36

La classe au titre de l'extension et telle métaphore au titre de la restriction imposent un renversement de la détermination : la foudre du divin, la foudre del'apoplexie, la foudre dela «présence» pour $\mathrm{H}$ ofmannsthal donnent, après renversement, ledivin dela foudre, del'apoplexie, dela «présence» et dégagent, ce faisant, un paradigme ignoré du dictionnaire. Selon la même perspective, il sembleurgent dedistinguer entre deux espèces de métaphores: (i) les métaphores paradigmatiques qui ont pour assiette les définitions des grandeurs, dont elles extraient un trait sémantique; deux grandeurs possédant chacune, par exemple, le trait/circularitél sont «métaphorisables»; les enfants exploitent sans ménagement cette possibilité; (ii) les métaphores syntagmatiques qui ont pour assiette les syntagmes possédant un élément commun qui devient dès lors le catalyseur d'une identiténon soupçonnée pour les deux éléments différents. L'«image» dans la définition proposée par P. R everdy ressortit à la deuxième espèce 37 .

Pour désigner le sujet selon la tonicité, nous empruntons à G. Bachelard le terme de sujet «tonalisé». D eux difficultés sérieuses se font jour: (i) les termes «sidéré» et «tonalisé» sont spécifiques pour autant qu'ils désignent des sujets affectés par des val ences extrêmes; pour poser la géné ricité, nousavançons, faute de mieux, les expressions: «sujet selon la vitesse» et «sujet sel on la tonicité»; (ii) l'intensité sémiotiqueétant, fort probablement, leproduit dela vitesse par la tonicité, comment atteindre chacun de ces sujets selon sa caractéristique? une possibilitéde solution apparaît: si le tempo et la toniciténe sont pas suspensibles, puisqu'ils sont le tuf même de notre devenir, ils sont cependant neutralisables selon I'acception structurale de ce terme, ce qui correspondrait àl'uniformitépour letempo: [ni accélération +ni ralentissement], et pour la tonicitéà la stabilitétonique: [ni augmentation + ni diminution]. Pour cette sousdimension, la stabilité demeure distincte de l'équanimité, laquelle admet certes le trait /stabilitél, mais pour des valences toniques faibles.

Puisquenous devonsà G. Bachelard leterme de «tonalisation», il est conséquent de le suivre dans sa description. Lesujet «tonalisé» présente, sous bénéficed'inventaire, deux caractéristiques: (i) ce sujet est un sujet enthousiaste:

[...] au lieu de chercher la qualité dans le tout de l'objet, commele signe profond de la substance, il faudra la chercher dans l'adhésion totale du sujet qui s'engage à fond dans ce qu'il imagine ${ }^{38 ;}$

(ii) le sujet «tonalisé» apparaît comme le gardien de l'excès, c'est-à-dire lereprésentant d'uneforme de vie plausiblequ'il est possible de situer d'un point de vue sémiotique: avec le recul que procure la durée, il apparaît que la sémiotique greimassiennea d'une part démonté et démontréla nécessité interne de la narrativité du modèle proppien, d'autre part généralisé ce modèle. T outefois, pour les sciences dites hu- 
maines, la nécessité n'emporte pas l'universel, mais son partage. D ela narrativité proppienne, nous aimerions souligner qu'elleintervient «en aval », c'est-à-direqu'elleassiste à la survenue du manque, puiselle entreprend sa résolution, si bien qu'on pourrait lui faire remarquer qu'elle $a$, en fin decompte, pour objet son propreaveuglement. Examinéà ce seul point devue, le sujet tonalisé semble soucieux d'intervenir «en amont »: il n'intervient pas après, mais avant; il ne met pas fin au manque, mais s'efforce de le prévenir:

Ainsi lesenscommun nes'y trompe pasqui répète, suivant le poncif, quelesvrais poètesnousfont «vibrer». M aissi cemot a un sens, il faut préci sément queletrop rappellelepas assez, et que le pas assez soit tout de suite comblé par le trop. 39

D ans la mesure où Bachelard fait, dans le même chapitre, une allusion rapide à $\mathrm{La} N$ aissance de la tragédie de $N$ ietzsche, nous sommes fondé à dire que le sujet tonalisé n'est pas un étranger pour l'«exalté dionysiaque».

Portrait du sujet équanime

Le sujet équanime, dont le Rousseau des Rêveries nous paraît un bon représentant, doit, jusqu'à l'administration du démenti, reproduire la division de l'intensité en sousdimensions. Eu égard au tempo, le sujet équanime s'avère un sujet selon la lenteur, sinon le repos; il a pour objet la permanence, la persistance, pour autant qu'elle repousse toute détermination; le sujet sel on la lenteur vise le miracle - oublié- del'allongement, dela perpétuation du temps 40 : «D e quoi jouit-on dans une pareille situation? De rien d'extérieur à soi, de rien sinon de soi-même et de sa propre existence, tant que cet état dure on se suffit à soi-même comme Dieu ». Ainsi que Rousseau l'indique, ce ne sont pas les noms et les verbes qui guident le sens, mais ici la conjonction de subordination tant queque L $\mathrm{M}$ M icro-Robert traduit par aussi longtempsque. C'est d'ailleursen vertu d'une «détermination» (H jelmslev) authentiquement grammaticale quela duréeau titre de variable «détermine» la lenteur au titre de constante. D u point de vue de la tonicité, le sujet équanimeest un sujet bien malaiséà cerner dans notre propre univers de discours. N ous admettrons, toujours à l'écoute de Rousseau, que le sujet équanime est un sujet quiet, «[jouissant...] de rien sinon de soi-même et de sa propre existence». O n songe bien sûr au «principe de N irvâna» cher à Freud, guère suivi sur ce point. Peut-être sommes-nous placé dans l'obligation de regarder en face un oxymoron particulièrement résistant: «le sentiment de l'existence dépouillé de toute autre affection». Comme si la sensorialité en cette extrémité ne délivrait pas une sensation, mais manifestait seulement sa propreet continue possibilité41. Un fragment des Cahiers nous le donne à entendre:

LeSilence- termequi représentela continuitédela fonction auditive. $L$ 'audition $=0$, maisl'audi bilitéexisteet est perçue - sous forme d'attente. Perception d'un pur pouvoir d'entendre - manque de réponse

Sentir qu'on ne sent pas. [... $]^{42}$

Si la forme du contenu propre au sujet quiet nous échappe en partie, Bachelard nous donne une indication précieuse de la forme de l'expression, quand il précise que le «psychisme» du sujet selon le repos est «involutif»:

Pris dans ses aspects humains, le repos est dominé nécessairement par un psychisme involutif. Le repliement sur soi ne peut pas toujours rester abstrait. II prend les allures de l'enroulement sur soi-même, d'un corps qui devient objet pour soi-même, qui se touche soi-même. II nous était donc possible de donner une imagerie de cette involution. ${ }^{43}$

La réflexivité dans le plan du contenu aurait pour manifestanteune morphologie bien caractérisée. Bien qu'il soit quelque peu contradictoire de préciser ce que l'on ne comprend pas, nous avanceronsl'hypothèsesuivante: I'existence sémiotique serait commutative, marquée par des retournements majeurs qui ne seraient saisis et démêlés qu'après coup parce que la syntaxe implicative a fait place subitement, sans s'annoncer, à la syntaxe concessive qui est la «mère» del'étonnement et de la surprise; ces configurations ne nous font pas sortir de la syntaxe: elles nous font changer de syntaxe! Q ue se passe-t-il exactement quand la tonicité s'amenuise? Selon la syntaxe implicative, il ne se passerait justement rien! il ne devrait rien se passer, mais, dans ces régions de l'espace tensif définies par des valences extrêmes, ce rien devient, par concession, quelque chose! Le fragment des $C$ ahiers le suggère: I'interruption de la fonction fonctionne 44 , fomentant un jenesaisquoi fascinant:

[...] mais bientôt ces impressions légères s'effaçaient dans I'uniformité du mouvement continu qui me berçait, et qui sans aucun concours actif de mon âme ne laissait pas de m'attacher au point qu'appelé par l'heure et par le signal convenu je ne pouvais m'arracher de là sans effort. 45 
Transition du sujet sensible vers le sujet praxique

Cependant le sujet sensible est forcément aussi un sujet praxique, un sujet qui intervient, c'est-à-dire qui enchaîne une visée à une saisie. Bachelard précise - en termes de valences- le paradigme:

Cette tonalisation du sujet, il semble qu'elle ait deux dynamiques différentessuivant qu'elleseproduit dansune sorte de tension de tout l'être, ou, au contraire, dans une sorte de liberté toute détendue, tout accueillante, prête au jeu des images finement rythmanalysées. 46 [...] Et ces rythmes sont lents, livrés précisément à celui qui veut les vivre lentement, en savourant son plaisir. 47

Le sujet «tonalisé» est un sujet qui, refusant la spécialisation sensorielle, promeut la transitivité d'un sens vers un autre, même s'il est très probable que cette transitivité n'échappe pas au conditionnement culturel, quel'«imagination», telle queBachelard la conçoit, doit impérieusement récuser: «Les correspondances sensibles apparaissent alors non pas à la base, mais aux sommets psychiques des différents sens» 48 .

Si maintenant nous envisageons le sujet équanime, il se présente comme un sujet que nous dirons en première approximation pénétrant; en effet, le verbe «pénétrer» en français syncrétise une morphologie spatiale désignant le passage canonique d'une extérioritéà une intériorité et un faire analytique: «parvenir à connaitre, à comprendred'une manière poussée». Le sujet pénétrant, opérant par tris successifs, est un sujet concentrant, tandis que le sujet «tonalisé» du faire synesthésique, opérant par mélanges, est un sujet étendant.

Commeces correspondances sont loin d'êtrecanoniques, il paraît préférable de convoquer la fonction sémiotique: le sujet selon l'atonie est un sujet qui s'accommode de la spécialisation sensorielle, tandis quele sujet sel on la tonicité exige qu' «un sens[soit excité] par un autresens». La tonicité au titre de manifestée qui toujours se dérobe a pour manifestante cette possibilité de transgression sensorielle.

Les distinctions proposées se laissent ainsi regrouper:

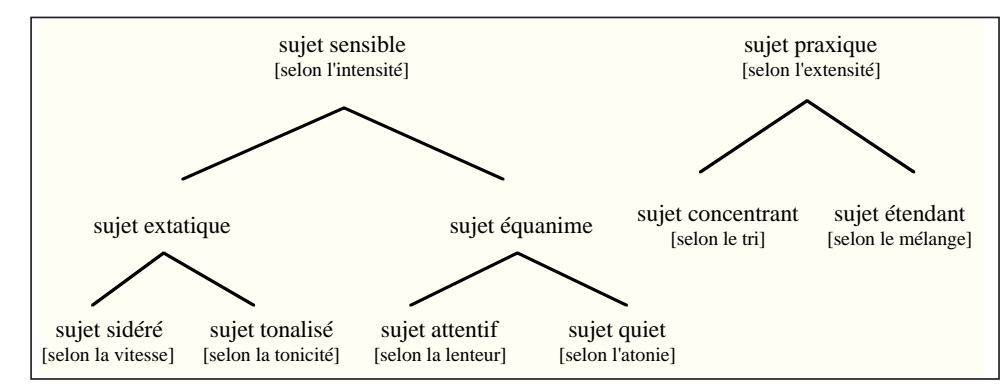

Profondeur du sujet savourant

La présence de la configuration du savourement dans la même citation va nous permettre de préciser le portrait du sujet selon l'atonie. Le M icro-Robert propose du verbe «savourer » la définition suivante: «1. M anger, boireavec toute la lenteur et l'attention requises pour apprécier pleinement. 2. Apprécier en prolongeant son plaisir $»$. Cette définition «sémiotisante» comporteau moins plusieurs enseignements profitables.

En premier lieu, les définitions des dictionnaires ont, à leur corps défendant, rapport aux valences tensives, sous une précision cependant: les valences peuvent, comme par faveur insigne, être flagrantes et, dans ce cas, l'analyse se hausse jusqu'à la transparence, c'est-à-dire à la paraphrase; ou bien être converties et, dans ce cas, l'analyse, comme suite de médiations, recouvreses droits; ainsi, pour fixer les idées, la première définition de «savourer» manifeste la valence de lenteur qui relève de la sous-dimension du tempo; la seconde définition envisage la durée et se propose, par implication, d'allonger le bref.

Le second point concerne la pertinence de la notion de valence; selon Saussure, la linguistique, telle qu'il la concevait, était caractérisée par le primat, sinon l'exclusivité, du point de $v^{2}{ }^{49}$ : au commencement est le point de vue, si bien qu'il n'y a pas degrandeurssubsistant indépendamment d'un point de vue déclaré, mais uniquement des grandeurs en attente d'un point devue. $D$ anslecas qui nous occupe, et sanstraiter ici la question au fond, nous admettrons que le sens doit être atteint en positivité, ce qui signifie que les contraires doivent, en dernièreinstance, êtresaisisen identité. Cetteidentité, nous la formulons en deux temps: en premier lieu, les valences appartenant à un gradient orienté, une valence est définiepar sa direction; en second lieu, cettedirection doit êtreen progrès, de sorte que la lenteur est dépréciée si elle est saisie comme vitesse décroissante, mais elle devient appréciable et appréciée si elle est vécue comme lenteur croissante, «majestueuse», mystérieusement gratifiante; ainsi, dans le poème en prose, L'Invitation au voyage, Baudelaire écrit:

O ui, c'est dans cette atmosphère qu'il ferait bon vivre, - làbas, où les heures plus lentes contiennent plus de pensées, où leshorlogessonnent lebonheur avec une plus profondeet plus significative solennité

La recette pour opérer un semblable retournement est simple, puisqu'elle consisteà substituer le plus demoins au moins de plus. 
Enfin, elle confirme que l'évolution diachronique est largement prévisible dèsquel'on y songeun peu: en intensité, le destin des valences intensives est décadent; imperceptiblement, le lexème se dirige vers son amenuisement, sinon son «extinction", comme le montre l'exemple bien connu du verbe «navrer» qui, dans la C hanson de Roland, est employé pour les blessures infligées à l'épée et qui aujourd'hui est d'un usage tout à fait convenu, c'est-à-dire creux ; de sorte que c'est moins de «disette» que la langue souffre, comme on le pensait au XvıII siècle, que de langueur; le lexème va, par projection métaphorique, de la restriction concentrantevers la diffusion étendante; en vertu d'un principe de constance à méditer, le lexème gagne en extensité ce qu'il perd en intensité, en accent 50 ; enfin, le passage de la première définition à la seconde virtualise, parcequ'il la précipite démesurément, la durée, en montrant comme instantané ce qui a pris «du » temps.

Instabilité discursive de l'objet

La versatilité du sujet est bien reçue dans notre univers de discours et G reimas lui-mêmea, si l'on ose dire, apporté à ce motif la caution de la sémiotique en posant que «les sujets d'état sont par définition dessujetsinquietset les sujets defaire, des sujets velléitaires» 51 , maisil en va à peu près de même pour l'objet.

\section{Vicissitudes de l'objet}

D ansla mesureoù nous accordons personnellement aux définitions des dictionnaires courants un crédit supérieur à celui qui leur est couramment accordé, il nous incombe d'envisager le couplage del'attention et dela lenteur dans la première définition du «savourement»: «1. M anger, boire avec toute la lenteur et l'attention requises pour apprécier pleinement». En premièreapproximation, la lenteur procure à l'attention la durée qui lui est indispensable: « 0 n voit quel'attention consisteà semodifier pour accroitreunecertaine durée»52. En second lieu, I'objet est tributaire del'ambiance propre au champ discursif à l'instant t. Les variations du tempo et dela tonicitédéterminent pour l'objet deux destins possibles inverses l'un de l'autre: (i) si l'intensité est croissante, l'objet entre en composition avec d'autres; telle sensorialitéspécialisée fait placeà la «bi-sensorialité»: tantôt «l'œil écoute» selon P.Claudel, tantôt l'«oreille voit», comme dans l'audition colorée devenueun jeu desalon dans la seconde moitié du XIX'e siècle; en un mot, l'accélération et la tonalisation seraient associatives; (ii) à l'inverse, le ralentissement et l'atonisation seraient dissociatifs et, selon une formule empruntée à V aléry, ils substitueraient une «pluralité» à une «unité», ou bien une «pluralité plus grande» à une «pluralité moindre»53. Contrairement à l'idée reçue, la labilité de l'objet n'est pas moindre que celle des sujets et l'apparaitre de l'objet entre ainsi dans la dépendance des caractéristiques momentanées du champ discursif:

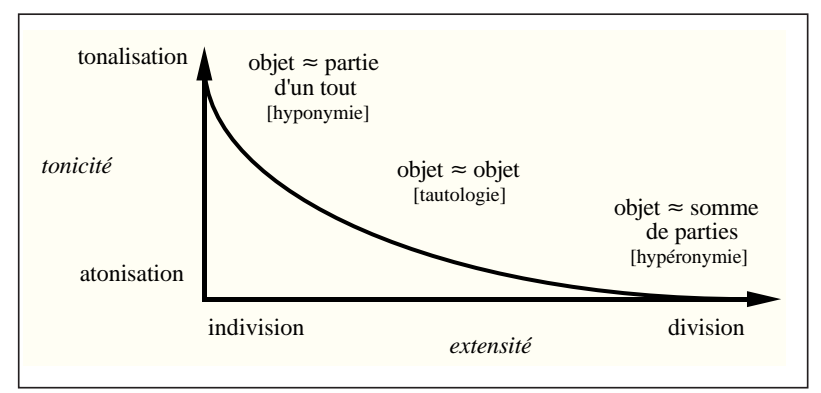

Si ces changements peuvent être considérés comme simplement «arithmétiques» dans le plan de l'expression, ils doivent être regardés comme commutatifs dans le plan du contenu et, en ce sens, «poïétiques»; à cet égard, on ne peut que donner raison à M erleau-Ponty quand il affirme la créativité de l'attention:

Ainsi l'attention n'est ni uneassociation d'imagesni le retour à soi d'une pensée déjà maîtresse de ses objets, mais la constitution active d'un objet nouveau qui explicite et thématise ce qui n'était offert jusque là qu'à titre d'horizon indéterminé. 54

Le paradigme de cette «nouveauté» est donc celui-là même del'événement et du repos: (i) selon l'événement, l'«image» en poésie, le «collage» en peinture, «la rencontre fortuite sur une table de dissection d'une machineà coudre et d'un parapluie!» (L autréamont) viennent occuper le champ discursif; (ii) selon lerepos, la compacitécirconstancielle, provisoire de l'objet, se retire et fait place à une stratification de nuances délicates, à une «verticalité» selon Bachelard:

[...] Edmond Jaloux nous fait sentir dans un vieux vin, dans un vin «dépouillé», «plusieurs bouquets superposés». En suivant l'écrivain, nous allons reconnaître toute la verticalité d'un vin. Ces «bouquets superposés», de plus en plus délicats, ne sont-ils pas à l'opposé d'un vin qui aurait un «arrièregoût»? Ce sont les bouquetssuperposésqui nousdisent unehauteur substantiellefaite d'un appel d'images desimageslesplusténues, lespluslointaines 55 
N ous pouvons achever le portrait sémiotique du sujet savourant:

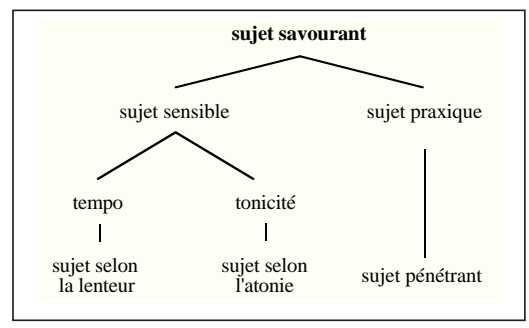

Tâches élémentaires du sujet praxique

Si le sujet selon l'intensité, le sujet sensible, est défini par les délicatesses qu'il ressent, le sujet sel on l'extensité, le sujet praxique, comme nous avons proposé de le dénommer, est caractérisé par les opérations dont il est capable et dont nous avons déjà touché un mot. Comme nous nous écartons quelque peu de notre propos, la lecture du texte deH ofmannsthal, nous nousen tiendrons aux propositions suivantes: (i) les opérations canoniques sur la dimension de l'extensitésont letri et lemélange, letri dégageant des valeurs d'absolu, lemélange, des valeurs d'univers; (ii) à partir des travaux de F.Bastide ${ }^{56}$ et d'A.J. Greimas ${ }^{57}$, nous introduisons, au titre de corrélats paradigmatiques des opérations, les grandeurs, et pour ces dernières elles-mêmes nous distinguons entre la cohésion, définie par son homogénéité, et la cohérence, définie par son hétérogénéité, l'une commel'autre stabilisées. Avant de poser les «intersections» constitutives des objets, il convient de définir d'un mot les opérations et les grandeurs: (i) le tri et le mélangeont affaire au commercedela division et del'indivision; (ii) la cohésion signifieici que la grandeur est «pure», qu'elle ne comprend que le même; la cohérence indique l'existence d'un partage entre le même et l'autre. Le rabattement des opérations sur les deux types de grandeurs mentionnées produit quatre configurations extensives en principe interdéfinies: le broyage, I'analyse, la solidification et l'amal game; les dénominations sont discutables en raison des syncrétismes toujours possibles; enfin nous avons porté entre crochets les catégories mentionnées par F. Bastide dans son étude. Soit:

\begin{tabular}{|c|c|c|}
\hline grandeurs & indivision $\stackrel{\text { tri }}{\rightarrow}$ division & $\begin{array}{c}\text { mélange } \\
\text { division } \rightarrow \text { indivision }\end{array}$ \\
\hline $\begin{array}{c}\text { cohésion } \\
\text { [même (1) / autre (0)] }\end{array}$ & $\begin{array}{c}\text { broyage } \\
\text { [pulvérisation] }\end{array}$ & $\begin{array}{l}\text { solidification } \\
\text { [massification] }\end{array}$ \\
\hline $\begin{array}{c}\text { cohérence } \\
\text { [même (n) / autre(1-n)] }\end{array}$ & $\begin{array}{c}\text { analyse } \\
\text { [concentration] }\end{array}$ & $\begin{array}{c}\text { amalgame } \\
\text { [expansion] }\end{array}$ \\
\hline
\end{tabular}

Sous les réserves suivantes, à savoir d'une part que d'autres catégories, telles que la récursivité et la réversibilité ne sont pas prises ici en considération, d'autre part que le tri et le mélange soient ici entendus comme termes géné riques, il est possible de décrire les activités ordinaires du sujet praxique: (i) le broyage, la trituration défont la consistance d'un «corps» en le réduisant à l'état dit poudreux; (ii) la solidification a pour programme de base le passage, ou le retour, del'état poudreux à l'état solide; elle demande souvent l'ajout d'un catalyseur, selon l'acception physique deceterme; (iii) l'analyses'attaqueà la cohérenced'un composé en vue d'établir la liste de ses ingrédients; (iv) l'amalgame rapproche et fond ensemble des grandeurs diverses, soit pour obtenir un composé nouveau, soit pour reproduire un composé existant et procurer ainsi un produit dit «de synthèse».

\section{Pour finir}

La sémiotique, guidée, contrainte par la «monotonie» (Propp) de son objet, atteint des récurrences, dégage des motifs de tous ordres, mais est-elle à même de changer, de «sublimer» ces motifs en raisons? La réponse est suspendue à la définition du concept de raison. D u point devuetensif, nous entendrons ici par raison le renvoi à un paradigme minimal associant et opposant des tensionsrésol uesà destensions non résolues Sous ce préalable sans doute hâtif, nous avançons l'hypothèse selon laquelle Lord C handos «souffre» de deux non-résolutions distinctes. La première, transitive, concernela relation entresystèmes, la seconde, réflexive, est interneau système optimal propreau monded'aujourd'hui.

\section{Tension transitive}

Lemonded'autrefois est un monde aristocratique, affirmant le prestige des valeurs d'absolu éclatantes et concentrées et corrélativement le mépris à l'égard des valeurs d'univers en principe mesurées et diffuses, mais disqualifiées comme «bourgeoises», «sentant la roture»... D u point devuemodal, le monde d'autrefois a pour assiette le parvenir de sorte que le sujet aristocratique, guidé par I'«honneur», doit mériter ce qu'il a reçu et s'employer de son mieux à augmenter une «gloire» célébrée, entre autres, par Corneille. Lemonded'aujourd'hui, dirigé par le survenir et la détonation de l'affect, se propose au fond de concilier l'éclat extrême propre aux valeurs d'absolu et la diffusion extrême propre aux valeurs d'univers, mais si l'éclat est bien réalisé, puis potentialisé: 
[...] je me détourne ensuite de ce lieu, en silence, puis, des semainesplustard, apercevant ce noyer, passe près delui avec un timide regard de côté pour ne pas effaroucher le sentiment laissé par le miracle qui souffle là, autour du tronc, ne pas chasser ces frissons autres que terrestres pesant encore dans le voisinage de ces broussailles, 58

La diffusion extrême demeure actual isée, le passage, décisif sur la dimension de l'extensité, du «je» au «nous»:

J'ai alorsl'impression quemon corpsest consituéuniquement decaractèreschiffrésavec quoi je peux tout ouvrir. 0 u encore que nous pourrions entrer dans un rapport nouveau, mystérieux, avec toute l'existence, si nous nous mettions à penser avec le coeur

est présenté comme une improbable éventualité. D e sorte quelemonde d'aujourd'hui finit par reproduire, aggravée, la structure discriminante propre aux valeurs d'absolu régissant le monde d'autrefois dont Lord Chandos a pris tacitement congé. À deux détails capitaux près: les valeurs nouvelles immanentes au monde d'aujourd'hui ne sont pas plus partagées que les valeurs traditionnelles gouvernant le monde d'autrefois. En second lieu, elles ne sont peutêtre pas partageables: les retrouvailles avec autrui supposent une concordance des expériences sensibles qui est difficilement pensable sans d'abord l'autorité d'une transcendance, dont Lord C handos dénie, sans s'y attarder, l'existence: «[...] ]'ai envie d'éclater en paroles dont je sais, les eusséje trouvées, qu'elles auraient terrassé ces chérubins auxquels je ne crois pas [...] »; sans ensuite la stabilisation de la fonction sémiotique:

Nul d'entre eux, devant sa porte et la casquette à la main, quand je passe à cheval, le soir, ne soupçonne [...] que mon regard [...] cherche, parmi tousces objets mi sérableset grossiers de la vie paysanne, celui, posé ou appuyé et n'attirant point I'œil, dont la formeinsignifiante, dont la nature muettepeut devenir la source de ce ravissement énigmatique, silencieux, sanslimite.

Le passage relatif àl'affrontement entre D omitius et C rassus va danslemêmesens: Lord C handos présente certes C rassus «comme un double de [lui]-même [...] par-dessus le précipice des siècles», mais ce passage en extensité de «un» à «deux» ne fait pas, loin s'en faut, un «nous».

\section{Tension réflexive}

La seconde tension est interne au système contraignant le monde d'aujourd'hui et elle justifie, nous semble-t-il, la précédente. La syntaxe caractéristique d'un système décadent, c'est-à-dire un système inauguré par la déflagration du survenir, alignetendanciellement deux macro-séquences:

sommation
[exclamative]
[désolution

Comme les termes adoptés le suggèrent, dans la perspective d'une prosodisation du contenu, la sommation 59 , correspond au moment, relativement mystérieux, de l'accent. D u point de vue tensif, l'accent est du ressort del'exclamation, laquelleapparaît commela limitedu discours 60 . Le M icro-Robert donne de «s'exclamer» la définition suivante: «Proférer des crisou des paroles en exprimant spontanément uneémotion, un sentiment». Le «cri »et la «parole», disjoints sous tel régime affectif, commutent I'un avec l'autre, commesi l'un - sous certaines circonstances reconnues comme des conditions - valait l'autre. Les prédicats communément admis de l'accent correspondent point par point aux catégoriestensives et l'on est en droit deconsidérer que l'accent les sature:

\begin{tabular}{|c|c|c|c|}
\hline \multicolumn{2}{|c|}{ intensité } & \multicolumn{2}{c|}{ extensité } \\
\hline tempo & tonicité & temporalité & spatialité \\
$\downarrow$ & $\downarrow$ & $\downarrow$ & $\downarrow$ \\
soudaineté & tonalisation & brièveté & localité \\
\hline
\end{tabular}

La sommation, laquelle confère enfin à l'exclamation sa portée sémiotique, opère une syncope de la discursivité «prosaïque». Lord C handos insistesur lefait que son «aphasie» est double: elleest contemporaine del'extase, puisqu'il qualifie son «ravissement» d' 'énigmatique, silencieux, sans limite», mais la résolution expectée, la reprise par la parole n'advient pas dans la mesure où la sommation euphorique n'a pas été potentialisée:

M aisquand cet étrangeenchantement m'abandonne, jene sais plusrien à son suje; ; jenepourraispas davantagealorsexpliquer au moyen de paroles raisonnables en quoi consistait cette harmoniequi nous traversait, le monde entier et moi [...].

$M$ ais un peu plus loin, Lord C handos laisse entendre que, quand bien mêmela potentialisation adviendrait, les langues de référence ne lui seraient d'aucun secours, car il songe, après ou parallèlement à Baudelai re61, à «une langue dont 
pas un seul mot ne m'est connu, une langue dans laquelle les choses muettes me parlent [...]».

Toutefois, les choses sont un peu plus compliquées: tardive, nocturne et intermittente, la figure de $C$ rassus vient médiatiser la coupure entre un présent oublieux et une mémoire restauratrice:

L'image de Crassus est parfois présente, la nuit, dans mon cerveau commeune écharde autour de laquelle tout suppure, bat et bout [...] Ce sont également des vertiges, mais de ceux qui nesemblent pas, comme lesvertiges du langage, conduire dans l'immensité sans fond, mais pour ainsi dire en moimême et au sein le plus profond de la paix.

Lord C handos n'imagine pas, comme le narrateur de La Recherche, que la mémoire tant «volontaire» qu' «involontaire», même si les mérites de la seconde surpassent en intensitéceux dela première, puisse venir à bout del'oubli. Si l'on fait abstraction dela prétérition inhérenteà la fiction selon laquelle on a rarement aussi bien dit l'impossibilité de direunesolution decontinuitééloigneici l'un del'autre le [re]dire du sentir. Ce passage n'est pas non plus sans rappeler le fragment suivant d'U nesaison en enfer: : 'C 'est la vision desnombres. N ous allonsàl'Esprit. C'est très certain, c'est oracle, ce que je dis. Je comprends, et ne sachant m'expliquer sans paroles païennes, je voudrais me taire» 62 .

Comment interpréter d'un point de vue sémiotique la persistance decestensionsnon résolues ? En effet, l'accession au «plus profond de la paix » est un moment parmi d'autres marqués par l'«horreur», comme dans le cas du poison administréaux rats. N ous admettrons en première approximation que le paradigme élémentaire des formes et des destins de vie comprend des formes de vie définies par l'occupation d'une place unique dans l'espace tensif et des formes de vie définies par des déplacements, des allers et retours entre plusieurs places, ces places étant définies par les valences différentielles que, sous bénéfice d'inventaire, nous discernons. Lord Chandos vit non seulement leclivage entre le monde d'autrefois, devenu le monde de l'absence, et le monde d'aujourd'hui, mais si ce dernier s'avère certes un monde de la présence, c'est d'une présence «spasmodique ", «critique », en cesensque cette présenceest privée de son extensitétemporelle, à savoir la permanence. À l'opposé de ceux de Rousseau dans la Cinquième promenade, les vécus extatiques auxquels Lord Chandos accède sont - en vertu d'une déclinai son tensive autoritaire- vifs, aigus, inter- mittents, enfin épars. T out se passe commesi, en possession de cartes maîtresses, il ignorait la règle, la grammaire permettant de bien « jouer », c'est-à-dire de « gagner », en un mot d'être heureux.

\section{N OTES}

17. N ous retenons du M icro-Robert les indications suivantes: «1. Le fait de révéler (ce qui était secret). Information qui apporte des éléments nouveaux, permet d'éclaircir une question obscure. 2. Phénomène par lequel des vérités cachées sont révélées aux hommes d'une manière surnaturelle. 3. T out ce qui apparaît brusquement comme une connaissance nouvelle, un principe d'explication; cette prise de conscience. 4. Personne qui révèle soudain de grands talents». La quatrième acception est une «variété» de la première.

18. Selon P.V Valéry: «La vitesse de la pensée devrait être tenue comme aussi significativequecelledelalumière(laquellea mis de 1675 à 1905 environ pour être mise en valeur). Cette vitesse, propriété de la sensibilité, et qui est relative àd'autresperceptions, entrelesquellesellesesitue, joueraitun rôledansunevraie "théorie de la connaissance" »(1942) (dans Cahiers, tome 1, Paris, Gallimard, 1973, p. 1100).

19. E. D urkheim, LesFormesélémentairesdela viereligi euse, Paris, P.U .F., 1994, p. 285-286.

20. $\mathrm{D}$ ans la conclusion de son étude relative à la philosophie de $\mathrm{H}$ eidegger, G.Steiner citeun textedeC oleridgequi noussembleen résonanceavec lesvécus, avec les survenus de Lord Chandos: «As-Tu jamais élevé ton esprit à la considération del'EXIST ENCE, commesimpleacted'exister? N eT' 'es-T u jamais dit, pensivement, CELA EST, insoucieux en cet instant de savoir si un homme était là, ou unefleur, ou un grain de sable? En un mot sans référenceà telle ou tellemodalitéou formed'existence? Si T u esvraiment parvenu àcela, Tu auras senti la présence d'un mystère, qui a dû pétrifier Ton esprit de terreur ou d'émerveillement [...]». (G.Steiner, M artin H eidegger, Paris, Albin M ichel, 1981, p. 202.)

21. «La vitesse maxima dans le monde réel absolu, est celle du réflexe (celle de la lumièreest unepurenotion, uneécriture). M aispour l'hommeil nepeut rien ressentir plus rapide que son changement propre le plus rapide. C'est cette vitesse qu'il faut introduire dans les équations psychologiques universelles. La prendre pour unité. [...] Cette vitesse joue dans toutes nos pensées, elle est impliquée danstoutesnosidées- et il nepeut pas en être autrement». (Cahiers, tome, 1, op. cit., p. 805.)

22. P. Fontanier, LesFigures du discours, Paris, Flammarion, 1968, p. 123.

23. C'est nous qui soulignons.

24. J. Laplanche et J. B. Pontalis, D ictionnaire de la psychanalyse, Paris, P.U .F., 1981, p. 210.

25. L. H jelmslev, La Catégorie des cas, M unich, W. Fink, 1972, p. 128.

26. O u l'inverse, lorsque la montagne accouche d'une souris...

27. Freud présente ce concept comme un «décentrement» et comme un «transfert et déplacement des intensités psychiques des différentséléments. Ce processus est la partie essentielle du travail du rêve», dans L'Interprétation des rêves, Paris, P.U.F., 1973, p. 266.

28. E. Cassirer, Langage et M ythe, Paris, Les Éd. de M inuit, 1989, p. 29-30.

29. E. Cassirer, La Philosophie des formes symboliques, tome 2, Paris, Les Éd. de M inuit, 1986, p. 57.

30. J.-J. Rousseau, LesRêveriesdu promeneur solitaire, Paris, G arnier-Flammarion, 1964, p.102. La lecture conjointe des deux extraits de C assirer et de Rousseau dégage un point important, à savoir l'antagonisme affirmé comme non surmontable dela «présence» et dela temporalité: la «présence» se déploieaux dépens du temps: «M aiss'il est un état où l'âme trouveuneassietteassez solide pour s'y reposer tout entière et rassembler là tout son être, sans avoir besoin de rappeler le passéni d'enjamber sur l'avenir; où letempsnesoit rien pour elle, où le présent dure toujours sans néanmoins marquer sa durée et sans aucune trace de 
succession, sansaucun autresentiment deprivation ni dejouissance, de plaisir ni de peine, de désir ni de crainte que celui seul de notre existence, et que ce sentiment seul puissela remplir tout entière; tant que cet état durecelui qui s'y trouvepeut s'appeler heureux, non d'un bonheur imparfait, pauvreet relatif, tel que celui qu'on trouve dans les plaisirs de la vie mais d'un bonheur suffisant, parfait et plein, qui ne laisse dans l'âme aucun vide qu'elle sente le besoin de remplir». (I bid.) (C'est nous qui soulignons).

31. G. Steiner, M artin H eidegger, op. cit., p. 169-170.

32. M . H eidegger, Lettre sur I'humanisme, Paris, Aubier, 1977, p. 165

33. Voir C.Zilberberg, «Le schéma narratif à l'épreuve», Protée, vol. $21, n^{0} 1$, hiver 1993, p. 65-93.

34. Voir C. Zilberberg, «Pour introduirelefairemissif», dansRaison et poétique du sens, Paris, P.U.F., 1988, p. 97-113.

35. «"Être" p[ou]r une pensée, c'est gagner à la course - comme le spermatozoïde qui sera élu. Ainsi la vitesse sera facteur d'existence». P.Valéry, Cahiers, tome 1, op. cit., p. 1091.

36. E. D urkheim, Les Formes élémentaires de la vie religieuse, op. cit., p. 271. (C'est nous qui soulignons.)

37. Voir note 26. C oïncidence ou nécessité arcane, la définition del'image par P.R Reverdy correspond presque mot pour mot à la conception del'intuition en mathématique avancée par $\mathrm{H}$. Poincaré: «Parmi les combinaisons que l'on choisira, les plus fécondes seront souvent celles qui sont formées d'éléments empruntésà des domaines trèséloignés; et jeneveux pas direqu'il suffise pour inventer de rapprocher des domaines aussi disparates que possibles; la plupart des combinaisons qu'on formerait ainsi seraient entièrement stériles; mais quelques-unesd'entreelles, bien rares, sont lesplusfécondesdetoutes». Citépar A. D amasio, L'Erreur de D escartes, Paris, O.J acob, 1995, p. 243.

38. G. Bachelard, La T erreet les rêveries du repos, Paris, J. Corti, 1992, p. 81. 39. Ibid., p. 90.

40. Cassirer parle à propos de la religion taoïste de «survie infinie de l'identique», de«répétition illimitéedu même», dansPhilosophiedesformessymboliques, tome 2, op. cit., p. 154.

41. D ans son livre L'Erreur de D escartes, A. D amasio évoque ce qu'il appelle la «perception de l'état d'arrière-plan du corps» en des termes étrangement consonants avec ceux de R ousseau: «M aisjepensequ'il existeencoreuneautre sortedeperception del'état du corps, quejesoupçonned'avoir précédélesautres dansl'évolution. Jel'appelle perception del'état d'arrière plan du corps, parce qu'ellea trait à la perception d'un état de fond plutôt qu'à un état émotionnel. Autrement dit, il nes'agit pas de ressentir unegrandeémotion àlaV erdi ou une émotion cérébralisée à la Stravinski, mais plutôt un niveau minimal detonalité et derythme. En fait, il s'agit dela perception delavieelle-même, delasensation d'être. [... ] U n état d'arrière plan correspond, au contraire, àl'état du corpstel qu'il se présente entre des émotions» (op. cit., p. 195-196.). La définition de l'émotion proposée par A. D amasio : «[... ] ]'émotion résultedela combinaison deprocessusd'évaluation mentale, simplesou complexes, avec desréponsesàces processus, issues de représentations potentielles. Ces réponses s'effectuent principalement au niveau du corps proprement dit, se traduisant par tel ou tel état émotionnel du corps, mais elles peuvent aussi s'effectuer au niveau du cerveau lui-même [...]» (ibid., p. 183) permet d'envisager une conciliation du point de vue immanent et du point de vue transcendant ( $\mathrm{H}$ jelmslev), puisque lesvalencesintensivesdetempoet detonicitésont placées, non sansressemblance sur ce point avec le concept de pulsion en psychanalyse, que Freud place à la lisièredu psychiqueet du somatique. Cen'est pastout: en concordanceavec les thèses deV aléry relativesà la primautédela vitesse (voirinfra lesnotes 44 et 46), le tempo et la tonicité permettent au moi et au non-moi de «communiquer», tantôtclairement, àsavoir en synchronismel'un avecl'autre, tantôtconfusément, en asynchronisme

42. P. Valéry, Cahiers, tome 1, op. cit, p. 1155.

43. G. Bachelard, La Terre et les rêveries du repos, op. cit., p. 5.

44. D anslepremier chapitre deL a D ialectiquedela durée(Paris, P.U.F., 1993), $G$. Bachelard évoqueà plusieursreprisesle«non-fonctionnement delafonction» et l'«expérience positive du néant».
45. C'est nous qui soulignons

46. Ibid., p. 87.

47. Ibid., p. 90

48. Ibid., p. 87

49. «Ailleursil y a des choses, des objets donnés, quel'on est libredeconsidérer ensuite à différents points de vue. Ici il y a d'abord des points de vue, justes ou faux, maisuniquement despointsdevue, àl'aidedesquel son créesecondairement les choses». Cité par É. Benveniste, Problèmes de linguistique générale, tome 1 , Paris, Gallimard, 1967, p. 39.

50. La formule pessimiste de V aléry relative à la conscience: «La conscience faite pour défendre l'être le ronge plus tard» (Cahiers, 1894-1914, Paris, Gallimard, 1988, p. 169) peut êtreétendueàla métaphore: ellesignified'abord un accroissement appréciéd'extensité, puisquelelexèmemétaphorisant, au lieu d'être strictement réservé à une classe, vient s'appliquer à un terme étranger à cette classe, mais peu à peu la continuation de cet accroissement même vaut comme dilution: s'appliquant à tout et à tous, il finit par nes'appliquer à rien ni à personne.

51. A.J. Greimas, Du SensII, Paris, Les Éd. du Seuil, 1983, p. 102.

52. P. Valéry, Cahiers, tome 1, op. cit., p. 919.

53. Ibid., p. 918.

54. M . M erleau-Ponty, Phénoménologiedela perception, Paris, G allimard, 1983, p. 39.

55. G. Bachelard, La T erre et les rêveries du repos, op. cit., p. 91.

56. F. Bastide, Le Traitement de la matière, Actes Sémiotiques, IX, 89, 1987. F. Bastide met en avant quatre oppositions directrices: [amorphe vs structuré], [discret vscompact], [expansévsconcentré] et [simplevscomposé]; il noussemble quela première [amorphevsstructuré] est en retrait par rapport aux trois autres 57. A.J. G reimas, «La soupeau pistou ou la construction d'un objet devaleur», dans Du SensII, op. cit., p. 157-169.

58. C'est nous qui soulignons

59. V oirJ. FontanilleetC. Zilberberg, T ension etSignification, Liège, P. M ardaga, 1998, p. 78-88

60. Conformément àl'hypothèse toujours reçue selon laquelle les troubles, les dysfonctionnements sont révélateurs desfonctionnements, C assirer fait état des travaux - anciens- sur l'aphasie du neurologue anglais Jackson, dont les conclusions diffèrent sensiblement decellesqu'acru devoir formuler par lasuite R. Jakobson: «L'observation de ces maladies fait donc directement ressortir qu'il y a deux couches d'expression linguistique parfaitement distinctes et relativement indépendantes l'une de l'autre: la première dans laquelle ne se manifestent que des états intérieurs, la seconde dans laquelle sont «visés» et désignés des états de chose objectifs. Ce sont ces deux couches que Jackson opposecommelangage «inférieur »et langage «supérieur »(inferior and superior speech). Seules les expressions du langage supérieur possèdent une «valeur propositionnelle» proprement dite (propositional value). T out notre langage intellectuel aaffaireàdetellesval eursqui ledominentetl'occupent complètement; il ne sert pas à exprimer des sentiments et des émotions mais se rapporteà des objets et à des relations entre objets. Or c'est justement cette aptitude à former et à comprendre des valeurs propositionnelles, et non le simpleusage des mots, qui subit une atteinte grave ou une suppression totale dans les troubles aphasiques». (La Philosophie des formes symboliques, tome 3, Paris, Éd. de M inuit, 1988, p. 241.)

61. À la fin de la troisième pièce des Fleurs du M al, Élévation, Baudelaire

n'écrit-il pas: Derrière les ennuis et les vastes chagrins

Q ui chargent de leur poids l'existence brumeuse,

H eureux celui qui peut d'une aile vigoureuse

S'élancer vers les champs lumineux!

Celui dont les pensers, comme des alouettes,

Vers lescieux le matin prennent un libre essor,

- Qui plane sur la vie, et comprend sans effort

Le langage des fleurs et des choses muettes!

62. A. Rimbaud, E uvres complètes, Paris, G allimard/La Pléiade, 1954, p. 221 